\title{
Courbes P-Y des pieux isolés dans le sable. Détermination à partir de l'essai pressiométrique
}

\section{A. BOUAFIA}

A. LACHENANI

Université de Blida

BP 270

Route de Soumâa 09000 Blida, Algérie bouafia1@yahoo.fr
L'analyse de la réponse d'un pieu isolé à un chargement horizontal est souvent menée en pratique à la base des méthodes du module de réaction, appelées aussi méthodes des courbes P-Y. Ces dernières ont connu un essor mondial considérable suite au développement d'importantes recherches expérimentales et numériques. L'article se propose de présenter une synthèse des résultats de l'interprétation des essais de chargement latéral en vraie grandeur des pieux isolés instrumentés dans des massifs sableux, en vue de la définition des paramètres des courbes $\mathrm{P}-\mathrm{Y}$, à savoir le module initial de réaction et la réaction latérale limite, à partir des caractéristiques pressiométriques du sable. L'étude montre clairement que ces deux paramètres varient en puissance avec la rigidité relative globale du système sol/pieu. A la base de cette formulation, une définition pratique des courbes P-Y hyperboliques dans le sable est proposée. L'application de la méthode proposée à des essais de chargement en vraie grandeur et en centrifugeuse montre une très bonne concordance entre les prévisions de la méthode, en termes de déplacements et moments de flexion, et les observations expérimentales.

Mots-clés : courbes P-Y, pieu isolé, module de réaction, sable, pressiomètre, essai de chargement latéral.

undertaken on the basis of P-Y curves-based methods, which are widely used due to the development of important

experimental and numerical research works.

The paper is aimed at presenting the results of a synthesis of the interpretation of full-scale horizontal loading tests of single instrumented piles in sandy soils, in order to define the parameters of $P-Y$ curves, namely the initial lateral reaction modulus and the limit lateral soil reaction, in correlation with the pressuremeter test parameters.

This study clearly showed a variation of $P$-Y curves parameters as a power of lateral soil/pile stiffness, on the basis of which a hyperbolic shaped P-Y curves were proposed. The assessment of the proposed method by predicting the soil/pile response in full-scale tests as well as in centrifuge tests showed a very good agreement between the computed deflections and bending moments, and the measured ones.

Key words: P.Y curves, single pile, lateral reaction modulus, sand, pressuremeter test, lateral loading test. 


\section{NOTATIONS}

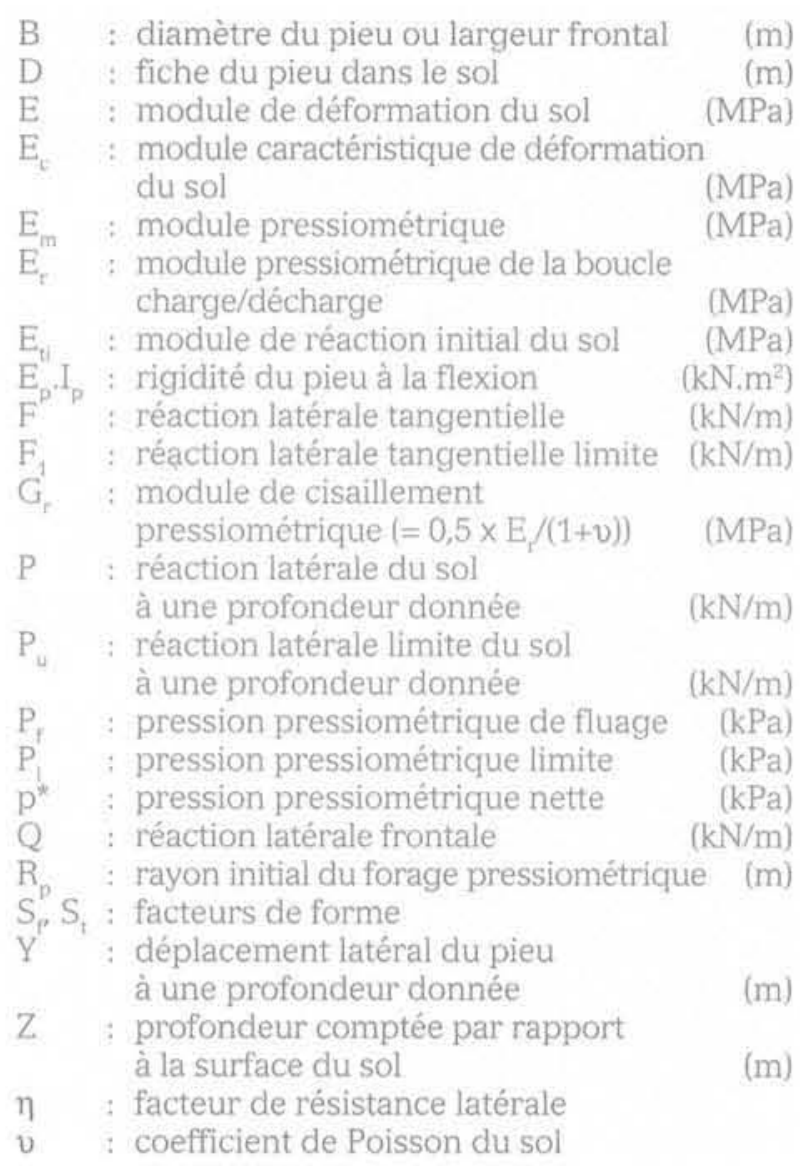

$(\mathrm{m})$

(MPa)

$(\mathrm{kN} / \mathrm{m})$

$(\mathrm{kN} / \mathrm{m})$

$\mathrm{kPa})$

$(\mathrm{kPa})$

$(\mathrm{kPa})$

(m)

v : coefficient de Poisson du sol de réaction était aussi appliquée pour l'analyse linéaire des déplacements du pieu en cas de massifs homogènes, en considérant le modèle d'une poutre sur appuis élastiques. Définies pour des configurations sol/pieu simples, ces méthodes simplistes s'avèrent insuffisantes pour tenir compte de l'hétérogénéité du sol ou de la non-linéarité prononcée de la réponse latérale du système sol/pieu.

En outre, les fondations sur pieux de certains ouvrages travaillant sous des charges latérales importantes sont dimensionnées selon les méthodes d'équilibre limite pour l'estimation de la résistance latérale du sol. Ces méthodes, reposant sur des schémas grossiers d'équilibre limite du sol autour du pieu, sont en général inspirées de la théorie de pression des terres sur les écrans rigides (Bouafia 1990; Bouafia et al., 1991; Bouafia, 1998).

L'essor considérable qu'a connu l'application des méthodes numériques en géotechnique et le développement des logiciels de calcul ont étendu les possibilités de modélisation, avec prise en compte d'une diversité de paramètres tels que la variabilité spatiale des propriétés du sol et la non-linéarité innée de la réponse de l'interface sol/pieu. Le concept de la courbe P-Y a été introduit pour décrire la relation entre la réaction $\mathrm{P}$ du sol et le déplacement $\mathrm{Y}$ du sol à une profondeur donnée, en définissant le module de réaction initial $E_{t}$ comme étant la pente initiale de cette courbe, et la réaction latérale limite $\mathrm{P}_{\mathrm{u}}$ en tant qu'asymptote horizontale correspondant aux grands déplacements latéraux. Dans plusieurs méthodes basées sur la théorie du module de réaction, ces deux grandeurs ont été liées aux caractéristiques mécaniques du sol, notamment celles mesurées aux essais in situ.

Le choix des paramètres de calcul conditionne la qualité de prévision de la méthode de calcul, et l'expérience montre que la clé d'une bonne prévision du comportement du pieu est un choix judicieux des paramètres de calcul plutôt que l'usage d'une méthode sophistiquée (Poulos et al., 2001).

Après un aperçu bibliographique des méthodes de construction des courbes P-Y, l'article se propose de présenter une synthèse des résultats de l'interprétation des essais en vraie grandeur des pieux instrumentés installés dans des massifs sableux assez homogènes, ainsi que la proposition de définition d'une courbe P-Y hyperbolique à partir des caractéristiques pressiométriques, à savoir le module pressiométrique $\mathrm{E}_{\mathrm{m}}$ et la pression limite $\mathrm{p}$

Enfin, on présente les résultats de validation de la méthode proposée en comparant les prévisions du comportement d'un pieu d'essai en vraie grandeur ainsi que celui d'un pieu prototype modélisé en centrifugeuse, aux observations expérimentales.

\section{2}

\section{Méthodes de construction des courbes P.y}

Les méthodes de construction des courbes P-Y ont connu une évolution considérable qui s'est manifestée le long de plusieurs décades. La première étude du pieu chargé horizontalement à la base de cette théorie semble être celle de Reese et Matlock (1956) en intro- 
duisant le concept du module de réaction, défini auparavant par Winkler (1867). L'interface sol/pieu est modélisé par une infinité de ressorts indépendants. La première génération des courbes P-Y comportait une courbe bilinéaire schématique d'un comportement élastoplastique de l'interface, La première droite, caractérisant les petits déplacements latéraux, a une pente égale au module de réaction latéral, et la seconde est définie par la réaction latérale limite. La non-linéaritê de réponse de l'interface est prise en compte selon un processus itératif d'équilibre du pieu sous les charges appliquées et les pressions latérales du sol. Le développement considérable des applications de l'informatique en géotechnique a accéléré la maturité des méthodes de calcul à partir des courbes P-Y.

L'essai pressiométrique est pratiquement le seul essai in situ pouvant donner une relation expérimentale entre les contraintes appliquées et les déformations des parois du forage. En outre, la similitude remarquable entre le mécanisme de réaction latérale frontale du sol autour d'une section du pieu, et celui de l'expansion d'une paroi de forage pressiométrique suggère que les deux mécanismes sont homothétiques, avec possibilité de passage de la courbe d'expansion pressiométrique à la courbe P-Y (Baguelin et al., 1978). Nombreuses sont les approches de construction des courbes P-Y à partir de l'essai pressiométrique et leur exposé même succinct sort du cadre de cet article. On se propose, dans ce qui suit, de présenter brièvement les méthodes les plus courantes.

\section{1}

\section{Méthode de Ménard, Gambin et Bourdon (1969)}

A notre connaissance, la méthode de Ménard, Gambin et Bourdon (1969), améliorée par la suite par Gambin (1979) est la première à la base de l'essai pressiométrique normal en forage préalable (PBPMT). Il s'agit d'une courbe trilinéaire dont la première droite a pour pente le module de réaction latéral de Ménard. Ce dernier est calculé à partir du module pressiométrique à l'aide de la formule de tassement dans la théorie pressiométrique, en assimilant le pieu à une semelle rigide infiniment longue, ayant une largeur B et dont le tassement est lui-même le déplacement latéral du pieu. La deuxième droite, définie depuis la pression pressiométrique de fluage $p_{f}$ jusqu'à la pression pressiométrique limite a une pente moitié de la première. La troisième est une droite horizontale correspondant aux grands déplacements avec une réaction latérale limite égale à p. multipliée par B.

Les caractéristiques pressiométriques intervenant dans la définition de cette courbe doivent être réduites le long d'une profondeur critique pour tenir compte d'un certain nombre d'effets tels que celui de la surface, dû à un remaniement en surface et une diminution du confinement vertical (Briaud, 1986).

Selon Ménard, la profondeur critique est de quatre fois le diamètre pour les massifs sableux (Frank et Jézéquel, 1989). Il est à signaler que les notions d'effet de surface et de profondeur critique échappent actuellement à toute analyse expérimentale directe.
Cette méthode a été intégrée dans les règles techniques françaises de conception et de calcul des fondations profondes (CCTG Fascicule 62, titre V, 1993), avec limitation de la réaction latérale limite à $\mathrm{p}_{\mathrm{f}} \mathrm{B}$.

Il est important de noter que l'analogie utilisée par Ménard entre le tassement d'une semelle continue rigide infiniment longue et la déflection d'un pieu présente des limites; il convient d'en préciser les conséquences sur l'évaluation du module de réaction latéral. En effet, les déflections du pieu sous les charges en tête mobilisent des réactions non uniformes le long du pieu, alors que le modèle de la semelle continue comporte des charges uniformes. En outre, cette analogie est limitée aux pieux rigides et ignore ainsi la rigidité à la flexion $E_{p} \cdot I_{p}$. Enfin, le modèle de semelle infiniment longue ignore l'élancement $\mathrm{D} / \mathrm{B}$ du pieu. La corrélation du module de réaction au module pressiométrique doit en principe tenir compte d'une rigidité relative $\mathrm{K}$. comme suit:

$$
k_{r}=\frac{E_{p} I_{p}}{E_{c} D^{4}}
$$

$E_{\text {e }}$ étant un module caractérisant la déformabilité du sol vis-à-vis du chargement latéral. Le modèle de Ménard fait partie de la catégorie des modèles élastiques en déformations planes disponibles en littérature, et qui sont résumés au tableau $\mathrm{I}$. On constate à partir de ce tableau que les corrélations de $E_{\text {t }}$ et $E$ ignorent complètement les paramètres mentionnés en équation (1). L'interprétation des essais de chargement de pieux montre toujours que dans le même site, les modules de réaction des pieux ayant des rigidités relatives $\mathrm{K}$ différentes, sont très différents, avec quasiimpossibilité de passage par calcul d'un pieu à l'autre, à partir d'un module de réaction unique (Bouafia 1990; Bouafia 2002a; Bouafia 2002b). L'expérience des essais sur modèles réduits centrifugés dans le sable confirme ce fait et suggère une variation du module de réaction en puissance de K (Bouafia 1990; Bouafia 2002c). Confrontée aux observations expérimentales sur pieux en vraie grandeur, l'analyse en petits déplacements par cette méthode est plutôt pessimiste (Frank 1984; Briaud 1986; Baguelin et Jézéquel 1972; Baguelin et al. 1990),

On note aussi que cette méthode ne tient pas compte de la réaction tangentielle mobilisée sur le fût du pieu, la contribution de cette dernière n'étant pas négligeable, particulièrement dans les massifs sableux, comme il sera vu ci-après. Belkhir et al. (1999) ont montré à travers le modèle de Pasternak, constitué d'une couche verticale incompressible travaillant seulement en cisaillement et intercalée entre le pieu et les ressorts de Winkler, une nette amélioration de la prévision des déplacements et moments fléchissants en tenant compte de la mobilisation des contraintes de cisaillement autour du pieu.

En outre, le seuil de mobilisation de la réaction limite, en termes de déplacements horizontaux, ne tient pas compte de l'influence de la rigidité du pieu. A titre d'exemple, ce seuil est entre 5 à $10 \%$ du diamètre pour un pieu de faible diamètre $(\mathrm{B}<60 \mathrm{~cm})$ quelle que soit sa rigidité.

Enfin, les prévisions de cette méthode en grands déplacements, comme l'enseigne l'expérience des essais sur pieux sont optimistes (Baguelin et al., 1990; Bouafia et Bouguerra 1995; Bouafia et Bouguerra 1996). 


\begin{tabular}{|c|c|c|c|c|}
\hline Modèle & Méthode & Paramètres requis & $E_{1} / E$ & Référencè \\
\hline $\begin{array}{l}\text { Disque se déplaçant latéralement } \\
\text { dans un cylindre }\end{array}$ & Élasticité & $\begin{array}{l}\text { Rayon Ro du } \\
\text { cylindre et v du sol }\end{array}$ & $\begin{array}{c}1,40 \text { pour } R o / B=10 \\
\text { et } v=0,33\end{array}$ & $\begin{array}{l}\text { Baguelin et al. } \\
(1977)\end{array}$ \\
\hline $\begin{array}{l}\text { Disque se déplaçant latéralement } \\
\text { dans un cylindre }\end{array}$ & $\begin{array}{l}\text { Éléments flinis } \\
\text { linéaires }\end{array}$ & $\begin{array}{c}\text { Ro fixé à } 10 \times \text { B } \\
\text { v fixé à } 0,33\end{array}$ & 2.25 & $\begin{array}{l}\text { Briaud ef al. } \\
\quad(1982)\end{array}$ \\
\hline $\begin{array}{l}\text { Plaque rectangulaire verticale } \\
\text { enfoule et chargée latéralement }\end{array}$ & $\begin{array}{l}\text { Élasticité } \\
\text { (équations de Mindlin) }\end{array}$ & $\begin{array}{l}\text { Elancement } \\
v\end{array}$ & $\begin{array}{c}1,90 \text { pour } \mathrm{D} / \mathrm{B}=10 \\
\text { et } \mathrm{v}=0,33\end{array}$ & $\begin{array}{c}\text { Douglas et Davis } \\
(1964)\end{array}$ \\
\hline $\begin{array}{l}\text { Plaque en déplacement latéral } \\
\text { dans un massif de sable }\end{array}$ & Elasticité & & 0.74 & $\begin{array}{l}\text { Terzaghi } \\
\text { (voir Oldham, 1986) }\end{array}$ \\
\hline Poutre clans un massif êlastique & Élasticitè & $E_{p}$ et B du pieu & $\begin{array}{c}0,4-1,0 \text { pour pieu en acier } \\
\text { et } B=0,5-1,0 \mathrm{~m}\end{array}$ & $\begin{array}{l}\text { Vesic (1961) } \\
\text { (voir Beikae } \\
\text { et Pyke, 1984) }\end{array}$ \\
\hline \multirow[t]{2}{*}{$\begin{array}{l}\text { Semelle continue } \\
\text { dans un sol élastique }\end{array}$} & Elasticité & $\begin{array}{l}\alpha \text { : coefficient de } \\
\text { structure du sol } \\
B \text {; diamétre du pieu }\end{array}$ & $0,9-1,1$ pour $B<0,6 \mathrm{~m}$ & Mènard (1971) \\
\hline & (synthèse des ètudes) & & $1,0-1,3$ & Bowtes (1997) \\
\hline Poutre dans un massif élastique & $\begin{array}{l}\text { Élasticité } \\
\text { (équations de Mindlin) }\end{array}$ & & 0,82 & Poulos (1971) \\
\hline
\end{tabular}

2.2

\section{Méthode de Dunand (1981)}

Cette méthode, se basant sur l'essai PBPMT, propose une courbe P-Y élastoplastique pour la réaction latèrale frontale. La pente initiale de la courbe est liée au module pressiométrique par le biais d'une méthode d'élasticité, et la réaction latérale limite est égale à $\mathrm{p}$ multipliée par B. Le concept de profondeur critique est introduit identiquement à la méthode précédente. Cette méthode est recommandée, selon son auteur, aux fondations sur pieux des pylônes électriques.

\section{3}

\section{Méthode de Briaud, Smith et Tucker (1985)}

Selon cette méthode, la courbe P-Y est la superposition des courbes Q-Y et F-Y décrivant les réactions frontale et tangentielle respectivement. CarayannaconTrezos et al. (1979) ont montré à travers une modélisation par éléments finis que la contribution de la réaction tangentielle augmente avec l'élancement de la section du pieu,

La courbe $Q-Y$ est construite directement à partir de la courbe d'expansion pressiométrique de l'essai normal (PBPMT) comme suit:

$$
\begin{gathered}
Q=S_{,} \cdot p^{*} \cdot B \\
Y=0,5 \times B \cdot \Delta R / R_{p}
\end{gathered}
$$

$\mathrm{S}$, est un facteur de forme égal à 1 pour une section carree du pieu, et à $\pi / 4$ pour une section circulaire. $\mathrm{R}$. est le rayon initial du forage pressiométrique et $\Delta R$ est l'augmentation du rayon du forage sous la pression p". L'équation (3) se base sur l'hypothèse d'une homothétie entre les déformations radiales dans l'essai pressiométrique et celles de la section du pieu en déplacement latéral.
La courbe de réaction tangentielle F-Y est bilinéaire, formée d'une portion initiale ayant une pente égale à 2.G. et une asymptote horizontale égale à $F$, fonction du frottement latéral limite $q_{s}$ mobilisé à l'interface sol/pieu, telle que:

$$
\mathrm{F}_{1}=\mathrm{S}_{1} \cdot \mathrm{q}_{5} \cdot \mathrm{B}
$$

$S$, est un facteur de forme pris égal à 2 pour les pieux carrés, et 1 pour les pieux circulaires. Selon Smith (1987), $q_{s}$ diffère peu de celui mobilisé lors d'un chargement vertical, et par conséquent peut ètre calculé par les méthodes usuelles de capacité portante des pieux.

Selon les auteurs de cette méthode, la confrontation de cette méthode aux données expérimentales collectées de 27 essais de chargement en vraie grandeur incluant une variété de pieux et sols, a donné une très bonne prévision des déplacements du pieu (Briaud, 1986).

Il est à noter que les réactions tangentielles limites sont mobilisées pour des niveaux de déplacements latéraux beaucoup plus petits que ceux pour la réaction frontale limite. Ce fait est analogue à celui de la mobilisation des frottements latéraux et de la résistance en pointe clans un chargement vertical.

\section{4}

\section{Méthode Baguelin, Jézéquel et Shields (1978)}

Cette méthode se base sur l'essai pressiométrique autoforeur (SBPMT) et se propose de déterminer la courbe $P-Y$ point par point, à partir de la courbe d'expansion pressiométrique comme suit:

$$
\begin{gathered}
P=\eta \cdot p^{*} \cdot B \\
Y=0,25 \times B \cdot \Delta V / V_{p}
\end{gathered}
$$

$\eta$ est appelé facteur de résistance latérale, tient compte du phénomène de profondeur critique et varie de 0,33 à 
3. $V_{p}$ est le volume initial du forage pressiométrique et $\Delta \mathrm{V}$ est l'augmentation du volume sous la pression nette $\mathrm{p}^{\star}$ (Baguelin, 1982).

\section{5}

\section{Méthode Robertson, Hughes et Campanella (1984)}

Cette méthode se propose de construire les courbes $\mathrm{P}-\mathrm{Y}$ d'un pieu foré à partir d'un essai pressiométrique normal (PBPMT) ou autoforeur (SBPMT), et celles d'un pieu battu à partir d'un essai au pressiomètre battu. Les formules (3) et (5) sont à utiliser avec un facteur $\eta$ égal à 1,5 pour les massifs sableux. La profondeur critique a été estimée à 4 diamètres dans cette méthode, et le facteur $\eta$ varie linéairement avec la profondeur depuis 0 en surface à 1,5 à la profondeur critique et au-delà (Robertson et al., 1985),

Une modélisation par éléments finis a été menée par Atukorala et Byrne (1984) pour étudier le déplacement latéral d'un disque rigide au sein d'un matériau élastoplastique de Coulomb, ainsi que celui dû à l'expansion d'une cavité cylindrique au sein du même matériau. II a été montré que le rapport des pressions latérales autour du disque et de la sonde pressiométrique, pour un déplacement donné, varie entre 1,4 et 1,7, soit en moyenne de 1,50. Ce résultat confirme la valeur de $\eta=$ 1,5 de la méthode précédente. Cependant, ce résultat est limité du fait que le comportement tridimensionnel du pieu a été ignoré.

Si on suppose que la pression limite et la réaction latérale limite correspondent à des déplacements latéraux infinis, on constate que les rapports $\mathrm{P} / \mathrm{P} . \mathrm{B}$, selon les méthodes vues précédemment, varient largement de 0,3 à 3,0, comme le montre le tableau II.

On se propose dans ce qui suit de présenter les principaux résultats obtenus lors de l'interprétation des études expérimentales des courbes P-Y, en corrélation avec l'essai pressiométrique (Bouafia, 2002a; Bouafia, 2002b). Des essais de chargement horizontal de 5 pieux isolés dans deux sites expérimentaux assez homogènes ont été menés par le Laboratoire central des ponts et chaussées en France de 1987 à 1989. Une synthèse de ces résultats permet en principe de dégager les principaux paramètres d'influence de la courbe de réaction latérale.

TABLEAUII Tableau comparatif des valeurs du rapport $\mathrm{P}_{\mathrm{u}} / \mathrm{p}_{1} \cdot \mathrm{B}$.

Comparison table of ratios $P_{i} / p_{1} B$.

\begin{tabular}{|c|c|c|}
\hline Méthode & $P_{u} / p, B$ & Remarques \\
\hline $\begin{array}{l}\text { Ménard et al. } \\
\text { Fascicule } 62 \\
\text { Dunand } \\
\text { Briaud et al. } \\
\text { Baguelin et al. } \\
\text { Robertson et al. }\end{array}$ & $\begin{array}{c}1,00 \\
0,50 \\
1,00 \\
0,83 \\
0,3-3,0 \\
1,50\end{array}$ & $\begin{array}{c}\text { Corrélation courante } p_{t}=p_{1} / 2 \\
\text { Pieu foré dans le sable } \\
\text { Au-delà de 4.B }\end{array}$ \\
\hline
\end{tabular}

\section{3}

\section{Résultats d'interprétation d'essais dans le sable}

\section{1}

\section{Sites et conditions géotechniques}

Le premier site, noté $S_{1}$, est localisé à la station expérimentale de Châtenay-sur-Seine, $70 \mathrm{~km}$ au sud-est de Paris. Une fosse profonde de $3,20 \mathrm{~m}$ et de $424 \mathrm{~m}^{3}$ de volume a été taillée dans un horizon de craie, remplie par du sable de Fontainebleau et saturée ensuite d'eau.

Des essais in situ au pressiomètre normal, pénétromètre statique à pointe électrique et pénétromètre dynamique aux normes européeennes ont été réalisés dans le massif sableux (Canépa, 1988). La figure 1 illustre les profils typiques obtenus de ces essais,

Le second site, noté $\mathrm{S}_{2}$, est localisé dans la commune du Rheu, $5 \mathrm{~km}$ au sud-ouest de Rennes. Il s'agit d'un terrain homogène issu des dépôts du pliocène et formé d'un massif de sable jaune ocre légèrement

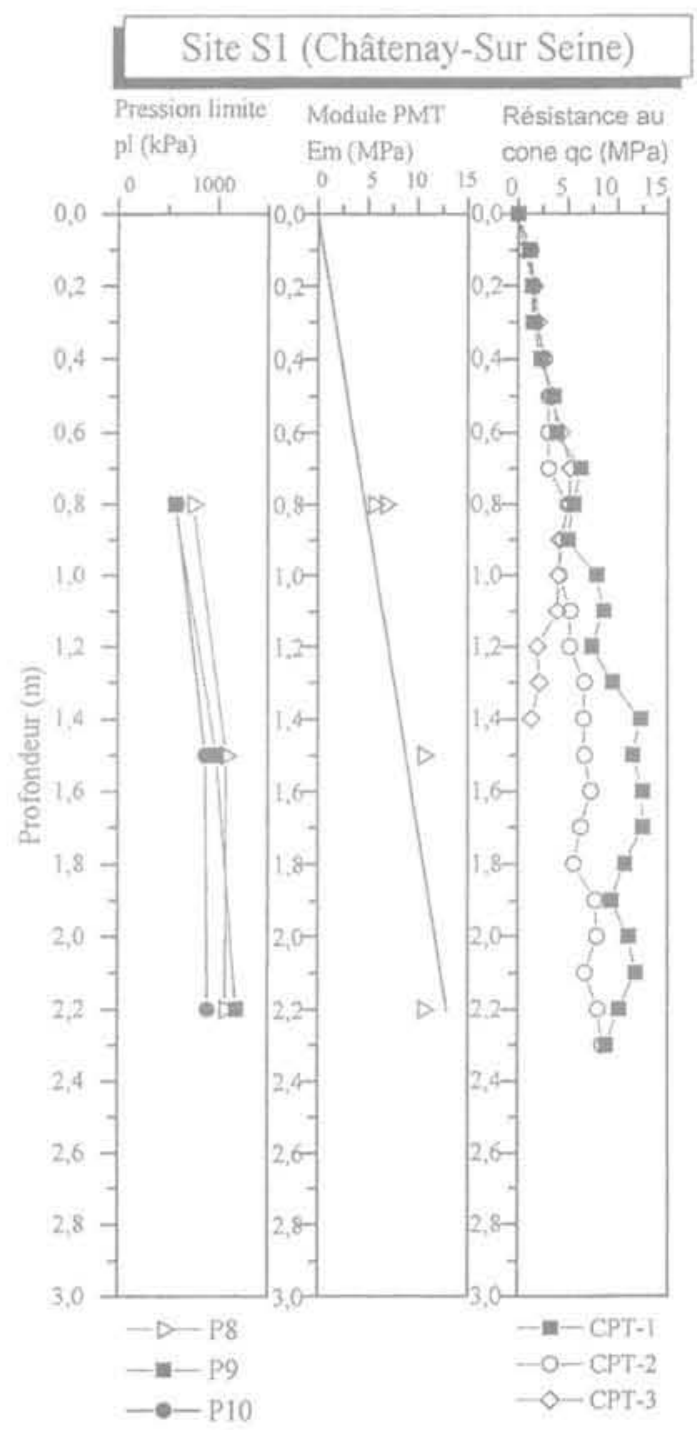

Fig. 1 Profils d'essais in situ au site de Châtenaysur-Seine.

In situ tests profiles in site Chảtenay-sur-Seine. 
humide pouvant former un talus vertical de plus de $10 \mathrm{~m}$ de hauteur. La nappe phréatique a été détectée à $10 \mathrm{~m}$ de profondeur. Au-dessus de la nappe, le sable a une teneur en eau de $8 \%$ et un degré de saturation de $31 \%$. Il a été possible d'extraire des échantillons à sec avec un carottier à hélice de $150 \mathrm{~mm}$ de diamètre jusqu'à $4 \mathrm{~m}$ de profondeur. L'indice de densité $\mathrm{I}_{\mathrm{D}}$ mesuré est de 66 \% (Jézéquel, 1988). Les résultats d'essais pressiométriques et pénétrostatiques, menés au droit des pieux d'essai, sont regroupés à la figure 2.

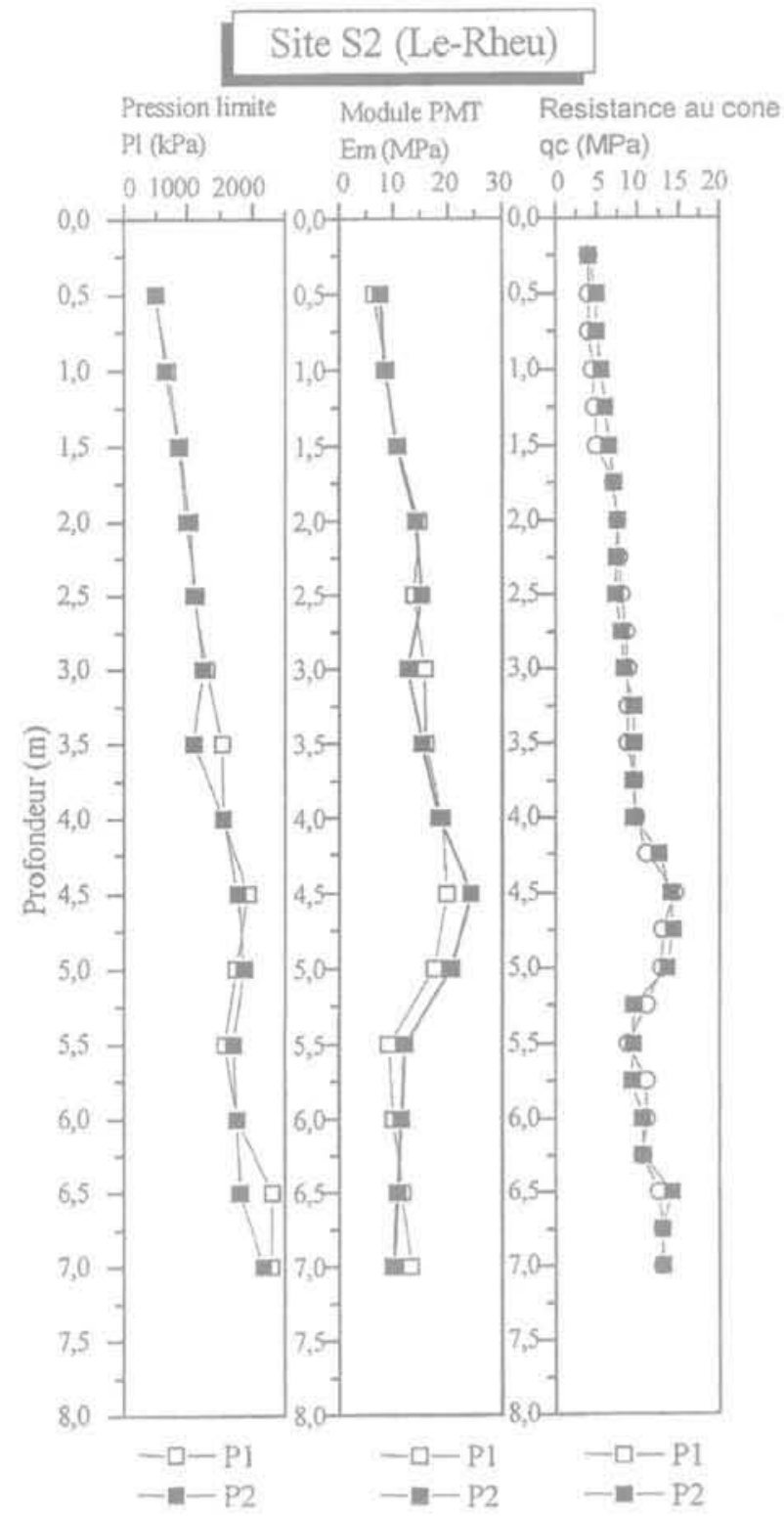

FIG. 2 Profils d'essais in situ au site du Rheu. In situ tests profiles in site Le Rheu.

\section{Pieux d'essais}

Les pieux utilisés sont des tubes en acier instrumentés par des jauges de déformation disposées par paires le long de deux génératrices diamétralement opposées. Le tableau III récapitule les différentes caractéristiques géométriques et mécaniques.

TABLEAU III Caractéristiques des pieux d'essai. Characteristics of test piles.

\begin{tabular}{|c|c|c|c|c|}
\hline Site & Pieu & $B(m)$ & $\mathrm{D} / \mathrm{B}$ & $\mathrm{E}_{p} I_{p}\left(\mathrm{kN} \cdot \mathrm{m}^{2}\right)$ \\
\hline S1 & $\begin{array}{l}\text { T5 } \\
\text { T10 } \\
\text { T15 }\end{array}$ & $\begin{array}{l}0,050 \\
0,100 \\
0,150\end{array}$ & $\begin{array}{l}14,2 \\
15,3 \\
15,3\end{array}$ & $\begin{array}{c}59,74 \\
868,9 \\
4331,6\end{array}$ \\
\hline S2 & $\begin{array}{l}\text { P1 } \\
\text { P2 }\end{array}$ & $\begin{array}{l}0,500 \\
0,900\end{array}$ & $\begin{array}{l}10,0 \\
5,50\end{array}$ & $\begin{array}{c}56370 \\
743600\end{array}$ \\
\hline
\end{tabular}

Trois pieux notés T5, T10 et T15 ont été testés sur le site $S_{1}$ et deux pieux notés $\mathrm{P}_{1}$ et $\mathrm{P}_{2}$ sur le site $\mathrm{S}_{2}$. Les élancements varient de 5,5 à 15,3 , Le pieu $P_{2}$ ayant un élancement de 5,5 peut être considéré comme un puits, avec tendance au comportement d'une fondation semiprofonde.

Les pieux tubulaires du site $\mathrm{S}_{1}$ ont été remplis par du coulis ciment-bentonite par injection par gravité à l'intérieur du pieu et remontée pour remplissage de l'espace annulaire entre le pieu et le forage (Jézéquel, 1988). Les essais de compression des éprouvettes du coulis à l'âge de 28 jours ont donné un module de déformation de $3500 \mathrm{MPa}$.

\section{3}

\section{Programme de chargement}

Chaque pieu a été soumis à une série d'incréments d'efforts statiques horizontaux appliqués en tête. La durée de chaque incrément est de 15 minutes au site $\mathrm{S}_{1}$ et de 2 heures au site $S_{2}$.

\section{4}

\section{Déroulement des essais}

Au site $S_{2}$, des perturbations de fonctionnement du vérin hydraulique ont modifié le programme initial de chargement en séries de 3 séquences de chargementdéchargement pour le pieu $\mathrm{P}_{2}$ et 2 pour le pieu $\mathrm{P}_{1}$. La réponse des jauges en surface du sol a été exploitée pour vérifier l'effort appliqué au pieu. En outre, des fissures ont été détectées au coulis pour des niveaux d'efforts élevés, ce qui a entrainé une chute de la rigidité à la flexion du pieu. A partir des déformations $\varepsilon$ mesurées en surface du sol, il a été possible de calculer la rigidité réelle du pieu à chaque niveau de chargement. Il a été constaté que le coulis s'est probablement fissuré au-delà d'un effort de 200 et $300 \mathrm{kN}$ pour les pieux $\mathrm{P}_{1}$ et $\mathrm{P}_{2}$ respectivement, ce qui a entraîné une chute de la rigidité de $11 \%$ pour le pieu $\mathrm{P}_{1}$ et de $20 \%$ pour le pieu $\mathrm{P}_{2}$. La relation $\mathrm{E}_{1}-\varepsilon$ obtenue au niveau de la surface a été supposée valable le long du pieu, ce quí a permis de calculer $E_{p} I_{p}$ de chaque section et d'en déduire le moment fléchissant (Bouafia, 2002a).

\section{5}

\section{Méthode d'interprétation}

Géométriquement parlant, la réaction latérale P du sol est au signe près, la courbure du moment fléchissant. Une légère incertitude sur la courbe des moments 
va engendrer par conséquent des variations importantes de la réaction latérale le long du pieu. Outre l'incertitude expérimentale sur les moments mesurés, les valeurs des réactions obtenues dépendent fortement de la courbe retenue pour le lissage des moments fléchissants expérimentaux. Par contre, les déplacements résultant d'une double intégration des moments sont beaucoup moins sensibles.

Le critère retenu pour le choix de la courbe de lissage est la vérification de l'équilibre statique du pieu sous les efforts en tête et la réaction du sol à $10 \%$ près (Bouafia et Garnier, 1991). Il a été constaté que les fonctions polynomiales de degré 5 et 6 pour les moments fléchissants des pieux des sites $S_{1}$ et $S_{2}$ respectivement, offrent un meilleur bilan d'efforts.

\section{6}

\section{Analyse des courbes P.y}

L'interprétation des données expérimentales pour chaque pieu a permis de construire les courbes de réaction à différentes profondeurs. La figure 3 illustre, à titre d'exemple, l'ensemble des courbes $\mathrm{P}-\mathrm{Y}$ du pieu $\mathrm{P}_{1}$. Une non-linéarité prononcée se manifeste même aux petits déplacements avec augmentation de la rigidité latérale avec la profondeur. En outre, au-delà d'un déplacement de $1 \%$ de B environ, on constate l'apparition d'un palier horizontal de réaction du sol.

L'ensemble des courbes de réaction ont été ajustées, au sens des moindres carrés, par une fonction hyperbolique de la forme:

$$
P=\frac{y}{\frac{1}{E_{t}}+\frac{y}{P_{u}}}
$$

Cette formulation est souvent utilisée pour décrire le comportement élastoplastique du sol (Duncan et Chang, 1970) ainsi que les courbes de réaction latérale (Reese 1971; Garassino 1976; Georgiadis et al., 1992).

Dans tous les cas étudiés ici, le coefficient de régression a été trouvé plus grand que $95 \%$ pour les profondeurs en deçà du point de déplacement nul, ce qui a permis de déduire les profils du module de réaction et de la réaction latérale limite dans cette zone. Au-delà, l'évaluation du module de réaction qui est en fait un rapport $\mathrm{P} / \mathrm{Y}$ aux petits déplacements, devient grossière vu les faibles valeurs des réactions et des déplacements.

Pour tous les pieux étudiés, le profil de $E_{\text {, }}(z)$ est pratiquement linéaire, ce qui en accord avec la distribution du module de déformation dans les milieux granulaires homogènes, dits massifs de Gibson. La figure 4 illustre un profil typique du module de réaction.

Pour valider la procédure d'interprétation des essais de chargement pour la construction des courbes P-Y, un calcul à rebours des pieux étudiés a été mené à partir de ces courbes. Ces dernières ont été introduites dans le programme PILATE (Pleu sous charges LATErales) développé au LCPC (Bangratz et Frank, 1985). Comme le montre l'exemple de la figure 5, une excellente concordance est à remarquer entre les déplacements calculés et ceux mesurés au site $\mathrm{S}_{1}$. Ce fait démontre la possibilité de décrire correctement le comportement du pieu, à tous les niveaux de chargement, à partir des courbes $\mathrm{P}-\mathrm{Y}$ ainsi construites.

\section{4}

\section{Synthèse des études des courbes P.y}

Les pieux d'essais étudiés sont caractérisés par différentes rigidités du sol et du pieu. On se propose de

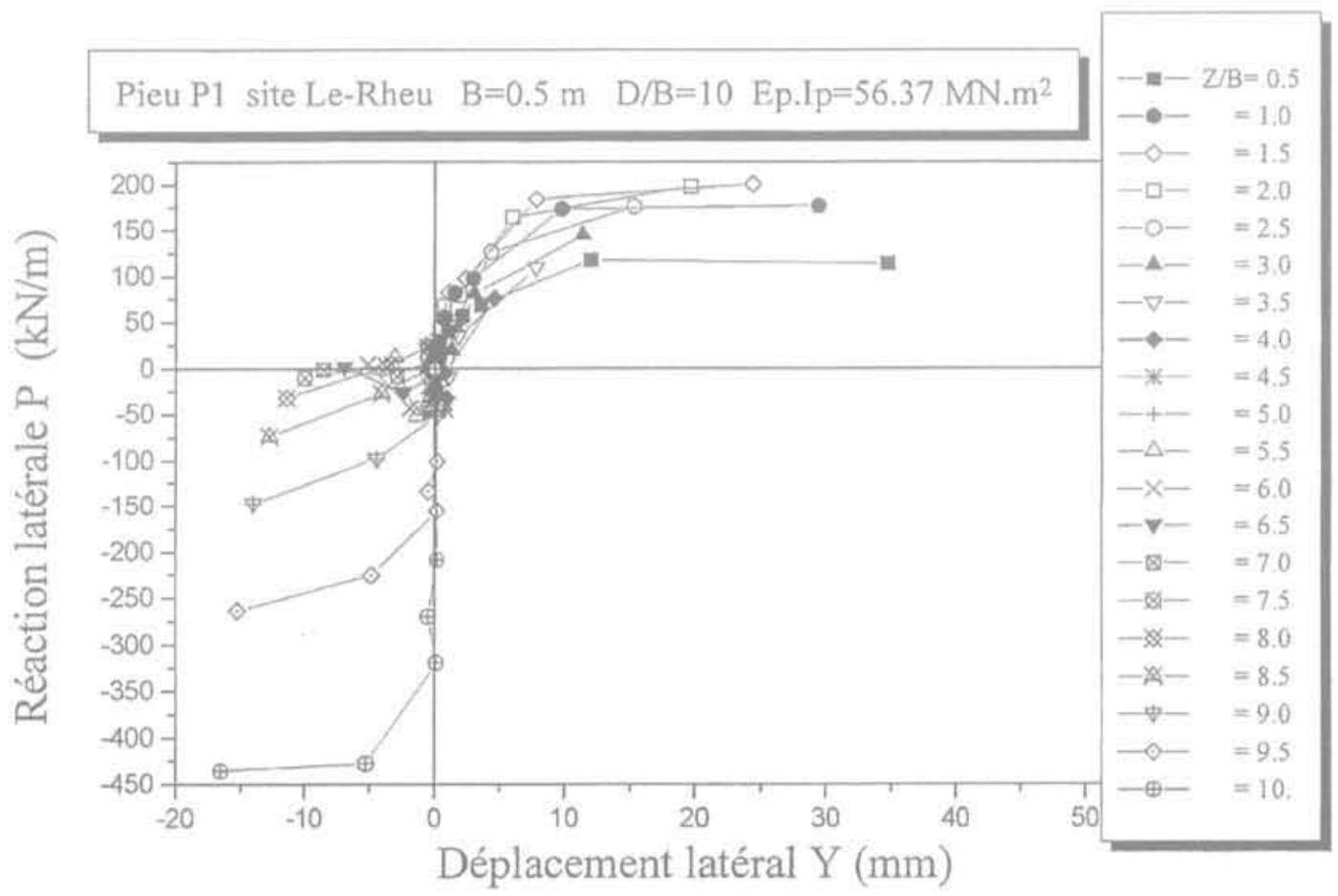

FG. 3 Courbes de réaction $\mathrm{P}-\mathrm{Y}$ du pieu $\mathrm{P}_{1}$. P. Y curves for pile $P_{1}$ 

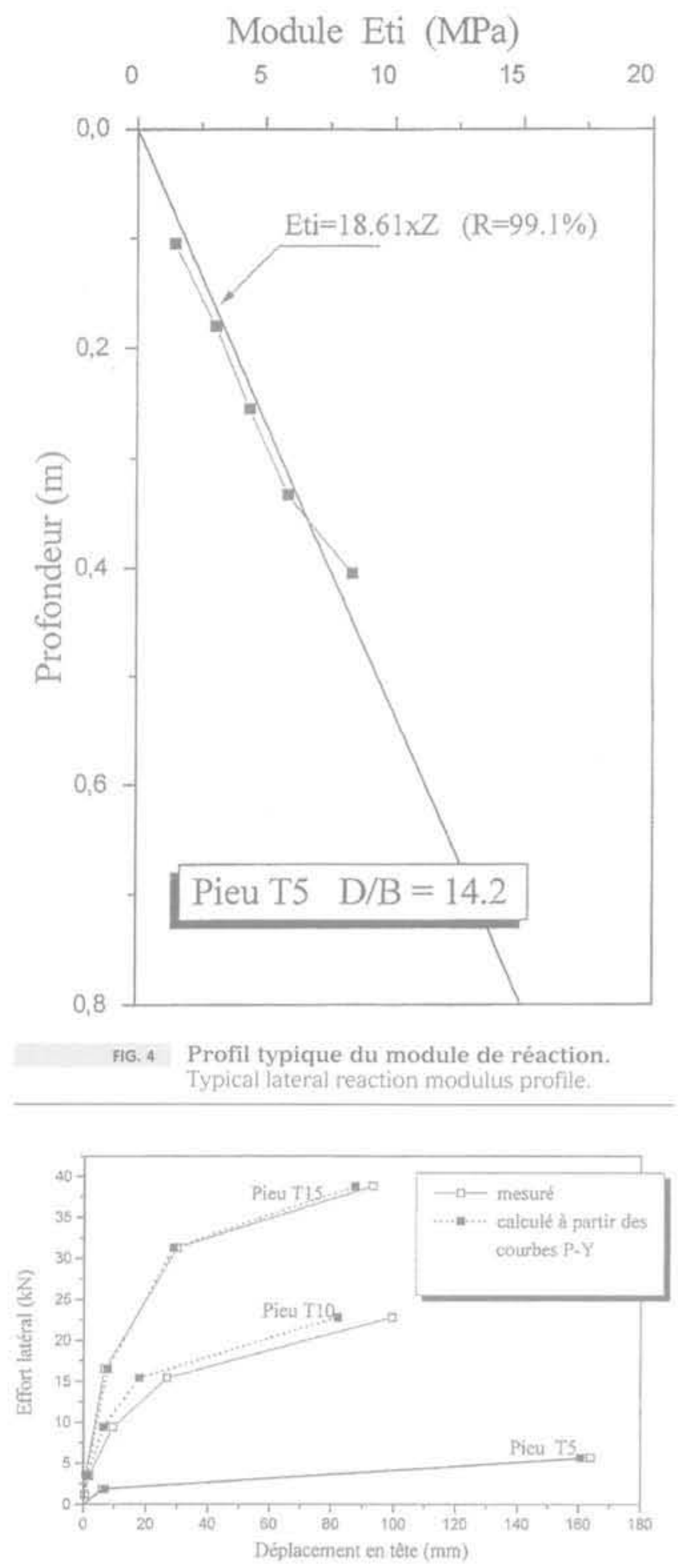

FiG. 5 Comparaison des déplacements calculés et mesurés au site $\mathrm{S}_{\text {. }}$.

Comparison between predicted and measured deflections in site $\mathrm{S}_{\text {. }}$.

définir la rigidité relative sol/pieu conformément à l'équation 1, en considérant comme module caractéristique de déformation $E_{\text {c }}$ du sol, une moyenne analytique des modules pressiométriques le long du fût du pieu, comme suit:

$$
E_{c}=\frac{1}{D} \int_{0}^{D} E_{m}(z) \cdot d z
$$

L'étude des valeurs moyennes du rapport du module de réaction initial au module pressiométrique, en fonction de la rigidité relative sol/pieu, montre d'après la figure 6 , une variation sensiblement linéaire dans un repère bi-logarithmique. Le point correspondant au Pieu $\mathrm{P}_{3}$, considéré plutôt comme un puits avec un élancement de 5,5 , est assez loin de cette corrélation linéaire, ce qui limite cette dernière aux pieux longs avec un élancement de plus de 10. Ainsi, il est possible de suggérer une variation du module de réaction initial en puissance de $\mathrm{K}_{\text {comme suit : }}$

$$
E_{t i}(z)=0,28 \times E_{m}(z) \cdot K r^{-12}
$$

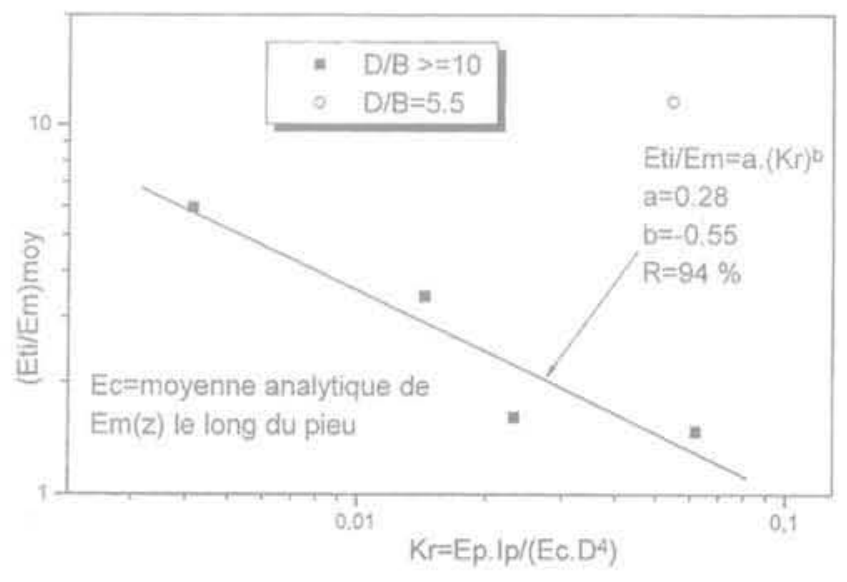

FIG.6 Variation du rapport $\mathrm{E}_{\mathrm{ii}} / \mathrm{E}_{\mathrm{m}}$ avec la rigidité relative.

Variation of ratio $E_{n} / E_{m}$ versus relative stiffness.

En outre, l'étude des valeurs moyennes du rapport $\mathrm{P} /(\mathrm{P}, \mathrm{B})$ pour les pieux étudiés, en fonction de la rigidité relative montre une augmentation en puissance, comme le montre la figure 7 :

$$
P_{0}(z)=3 \cdot P_{1}(z) \cdot B \cdot K_{f}^{12}
$$

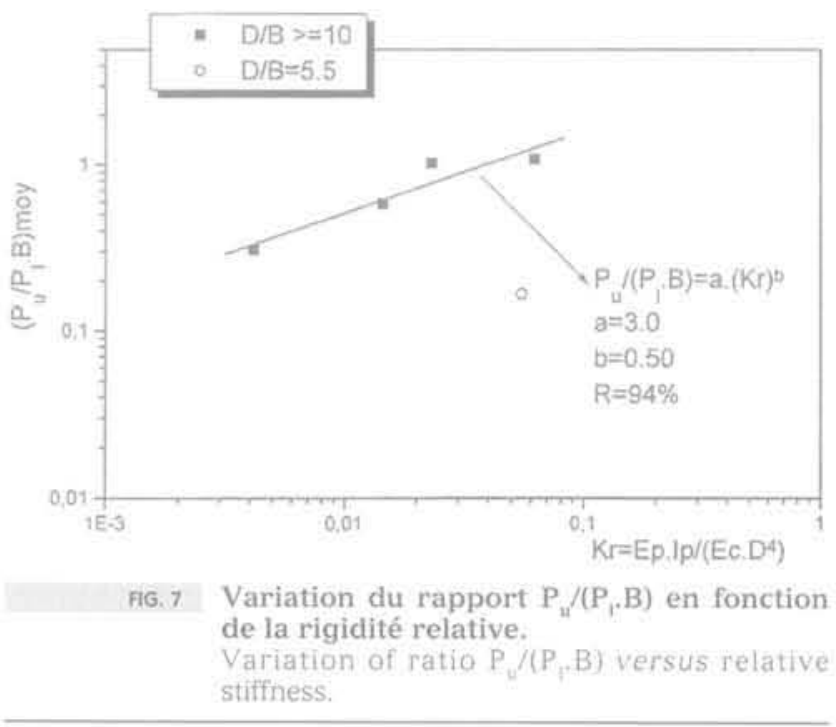

Cette relation simple montre qu'un pieu rigide mobilise une résistance latérale limite plus grande que celle pour un pieu souple, toutes choses étant par ailleurs égales. En outre, la réaction limite diminue avec l'élancement du pieu, contrairement aux relations courantes regroupées au tableau II, ignorant ce paramètre. 
Dans la marge de $\mathrm{K}$ caractérisant les pieux étudiés, soit de $10^{-3}$ à $10^{-2}$, le rapport $\mathrm{P}_{\mathrm{u}} /(\mathrm{P}, \mathrm{B})$ varie respectivement de 0,1 à 0,3 , ce qui est plus petit que les valeurs prescrites dans ce tableau. Quant au module de réaction latéral initial dans un sol donné, selon l'équation 9, il diminue avec la rigidité du pieu et augmente avec son élancement. Le rapport $E_{\mathrm{n}} / \mathrm{E}_{\mathrm{m}}$ varie de 9 à 3 , pour la marge de $K_{r}$ de $10^{-3}$ à $10^{-2}$ des pieux étudiés.

Les courbes P-Y hyperboliques définies par les équations 7, 9 et 10, présentent une approche simple de calcul du pieu chargé latéralement à partír de l'essai pressiométrique et il importe de tester sa qualité de prédiction.

\section{5}

\section{Test des prévisions de la méthode proposée}

Un calcul des pieux d'essai, quì ont servi à êtablir la méthode de construction des courbes P-Y hyperboliques, à été mené. La figure 8 montre une très bonne concordance entre les prévisions en termes de déplacernents, à partir des courbes P-Y, et les observations expérimentales. A titre de comparaison, la méthode du fascicule 62 a été appliquée au pieu $\mathrm{P}_{1}$ (Baguelin et al., 1990). La figure 9 montre qu'elle est légèrement optimiste en grands déplacements et la méthode proposée prévoit mieux les déplacements latéraux du pieu d'essai.

On se propose dans ce qui suit de modéliser le comportement d'un pieu d'essai en vraie grandeur décrit par Ruesta et Townsend (1997) au cours du projet du pont de Roosevelt en Floride. Le site est formé de deux couches submergées par l'eau à $2 \mathrm{~m}$ au-dessus du terrain naturel. La première couche est du sable lâche, épaisse de $4,0 \mathrm{~m}$, surmontant un horizon de sable cimenté. Le pieu est en béton précontraint avec un module $\mathrm{E}_{\mathrm{j}}$ de $34475 \mathrm{MPa}$, de section carrée de côté $0,76 \mathrm{~m}$, fichè à $14 \mathrm{~m}$ dans le sable, et installé par battage. L'effort latéral a été appliqué à $2 \mathrm{~m}$ au-dessus de la surface.

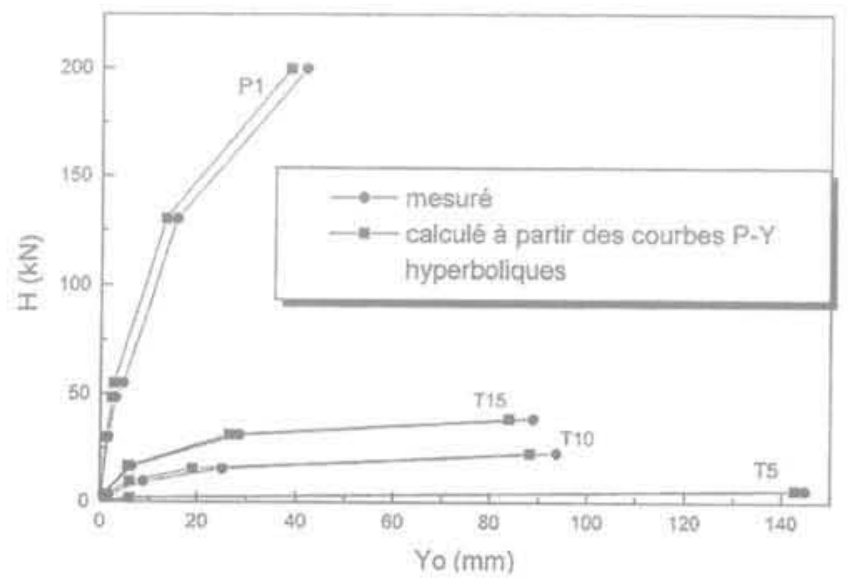

FIG, 8 Comparaison entre les déplacements en surface calculés et mesurés.

Comparison between predicted and measured surface deflections.

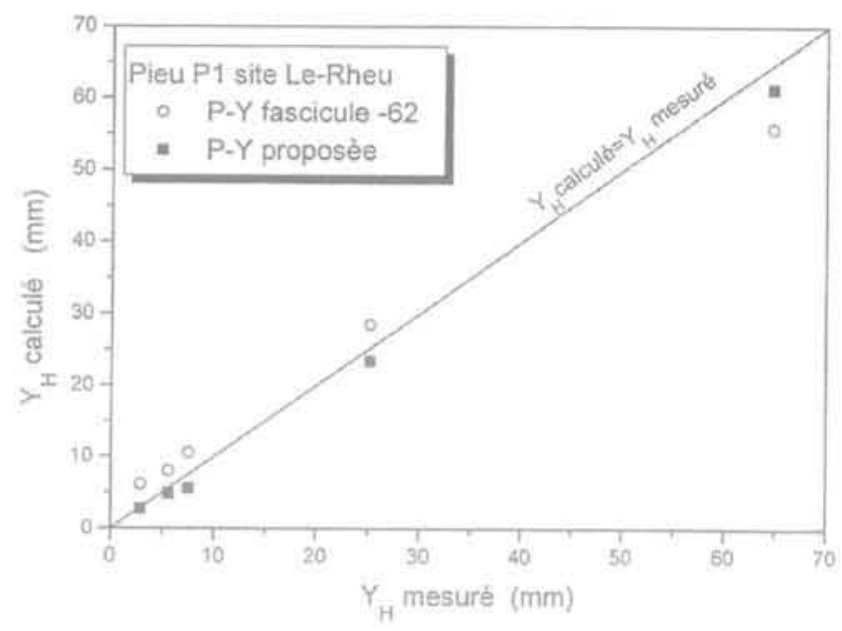

FG.9 Comparaison des prévisions à partir des courbes P-Y.

Comparison between P-Y curves - based predictions.

Au cours de l'essai, il a été constaté l'apparition des fissures dans le béton à partir d'un effort latéral de $200 \mathrm{kN}$. La rupture du matériau du pieu a eu lieu pour un effort de $320 \mathrm{kN}$.

D'après l'équation (8), le module caractéristique est de $61,3 \mathrm{MPa}$, et la rigidité relative selon l'équation 1 est. de $4 \times 10^{-4}$. Il s'agit en fait d'un pieu très souple. Le pieu a été divisé en 10 tranches et les paramètres suivants ont été calculés au milieu de chaque tranche, conformément aux équations (9) et (10):

$$
\begin{aligned}
& E_{v}(z)=20,9 \times E_{m}(z) \\
& P_{u}(z)=0,06 \times P_{i}(z) \cdot B
\end{aligned}
$$

La figure 10 montre une très bonne prévision des déplacements pour des efforts en deçà de $200 \mathrm{kN}$, seuil de fissuration et chute de la rigidité du pieu. En outre, selon la figure 11, les moments de flexion le long du pieu sont en bonne concordance avec les mesures à partir des jauges de déformation.

La méthode proposée a été aussi testée en étudiant un essai de chargement latéral d'un modèle réduit placé en centrifugeuse du LCPC. Ce modèle simule un pieu tubulaire prototype ayant un diamètre de $0,50 \mathrm{~m}$, une fiche de $5,0 \mathrm{~m}$, une rigidité à la flexion de $56650 \mathrm{kN} \cdot \mathrm{m}^{2}$, placé par forage dans un massif sableux

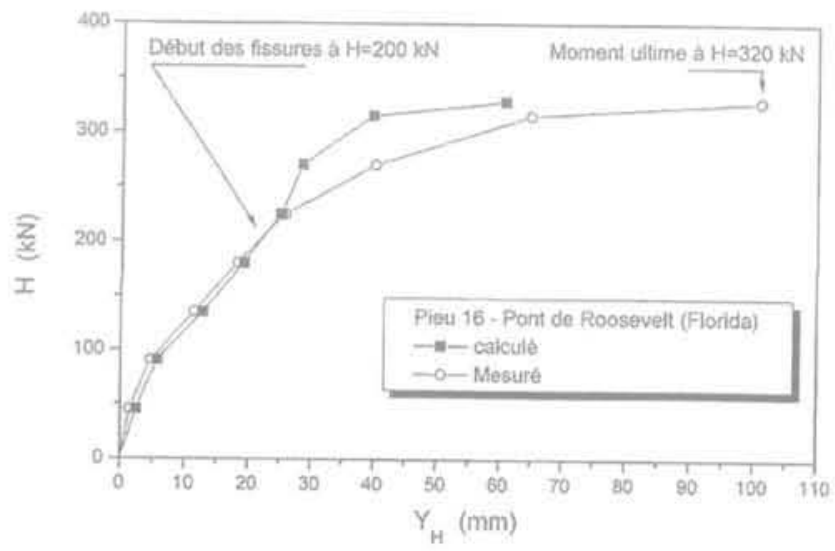

FIG. 10 Comparaison des déplacements en tête mesurés et calculés.

Comparison between measured and predicted top deflections. 


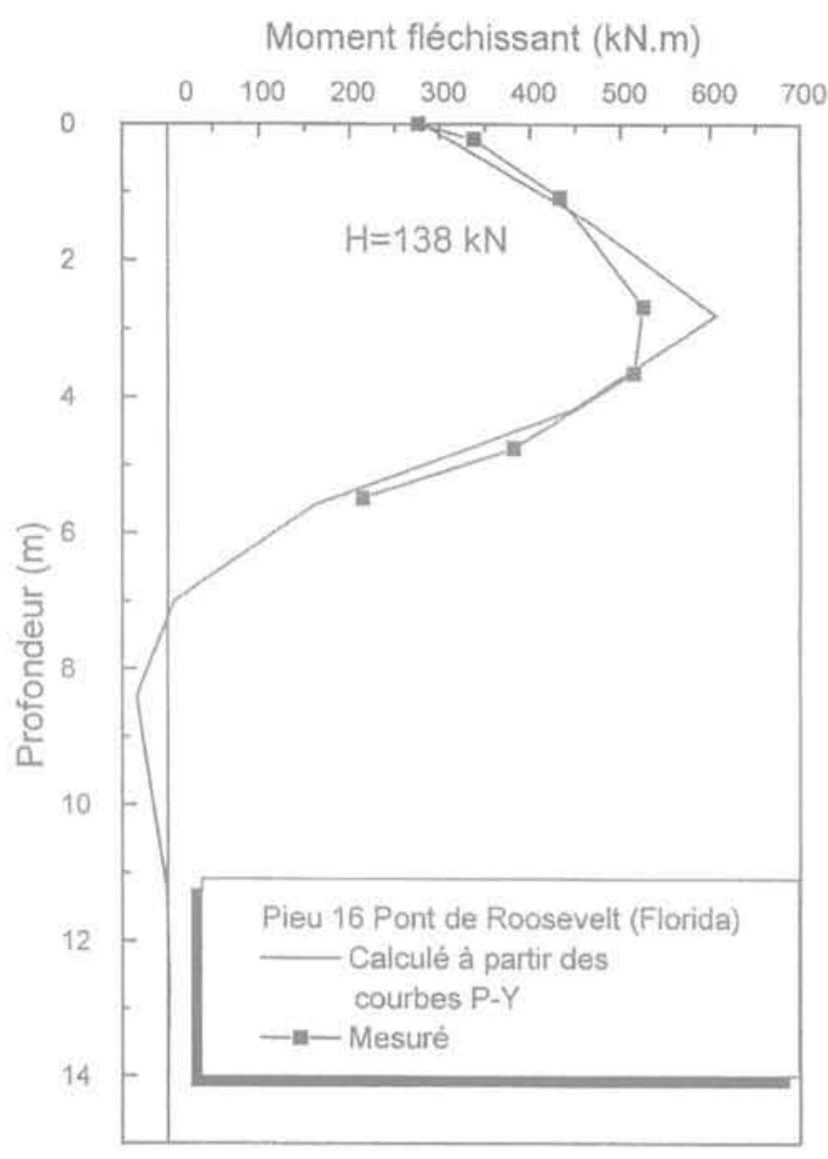

FIG.11 Comparaison entre les moments fléchissants calculés et mesurés.

Comparison between predicted and measured bending moments. homogène très dense. L'essai CPT a été réalisé avec un pénétromètre miniature embarqué en centrifugeuse et ayant un diamètre de $12 \mathrm{~mm}$. Pour définir les paramètres pressiométriques nécessaires à la construction des courbes $\mathrm{P}-\mathrm{Y}$, on admet en première approximation, que les corrélations PMT/CPT connues de ce sable in situ sont valables pour le sable en centrifugeuse, soient $\mathrm{q} / \mathrm{p}_{1}=7$ et $\mathrm{E}_{\mathrm{m}} \mathrm{q}_{\mathrm{c}}=1,5$ (Bouafia, 1990). Les calculs ont donné $K_{r}=4,8 \times 10^{-3}, E_{t} / E_{m}=5,3$ et $P /(P . B)=0,20$. Selon la figure 12 , dans le cadre de cette hypothèse de corrélation, les déplacements latéraux sont en bonne concordance avec les mesures extrapolées à l'échelle prototype.

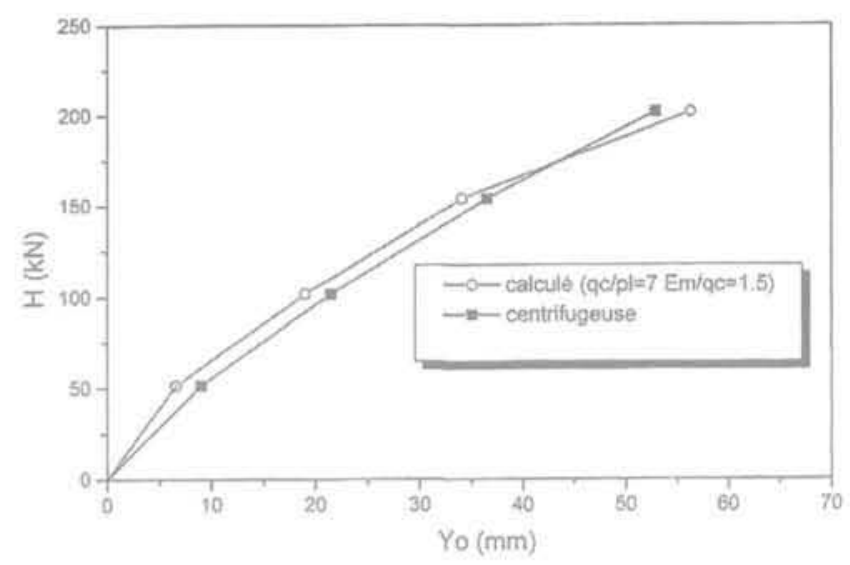

FiG.12 Comparaison des déplacements mesurés en centrifugeuse et calculés.

Comparison between measured deflection in centrifuge and predicted.

\section{Conclusion}

L'étude du comportement de 5 pieux expérimentaux sous charges latérales dans deux massifs sableux assez homogènes a permis de construire les courbes de réaction latérale $\mathrm{P}-\mathrm{Y}$ selon une procédure d'interprétation des données expérimentales. Un calcul à rebours a validé cette procédure, et une synthèse des résultats a montré que les paramètres de la courbe de réaction hyperbolique, à savoir le module de réaction initial et la réaction latérale limite dépendent, outre des caractéristiques pressiométriques, de la rigidité relative sol/pieu. Cette dernière fait intervenir un module de déformation caractéristique du sol fonction du module pressiométrique.

La méthode proposée pour la construction des courbes P-Y à la base de l'essai pressiométrique a été testée avec succès en recalculant les pieux d'essai. En outre, elle a pu prévoir correctement le comportement d'un pieu réel soumis à un essai de chargement en vraie grandeur dans un sol sableux, ainsi que celui d'un pieu prototype simulé en centrifugeuse. Cette méthode est à étalonner sur un nombre plus représentatif d'essais sur pieux dans le sable.

\section{REMERCIEMENTS}

Les auteurs sont reconnaissants à la direction du Laboratoire central des ponts et chaussées pour les avoir autorisés à utiliser les données des essais de chargement des sites expérimentaux de Châtenay-sur-Seine et Le Rheu dans le cadre de cet article. 
Atukorala U., Byrne P.M.- « Prediction of P-Y curves from pressuremeter tests and finite element analyses $n$. Soil Mechanics series $n^{\circ} 66$, Dept. of civil engineering, University of British CouIombia, July 1984, 34 p.

Baguelin F. - "Règles de calcul des fondations à partir de l'essal au pressiomètre autoforeur m. Comptes rendus du symposium La pressiométrie et ses applications en mer, Paris, 19-20 avril 1982 , éditions LCPC-IFP, p. 359-371

Baquelin $F$. Jézéquel J.F. - "Étude expérimentale du comportement des pieux chargés latéralement $x$. Annales de I'TTBTP, n 297, 1972, p. 155-200

Baguelin F, Frank R., Said Y, - Theoretical study of lateral reaction mechanism of piles 》n Geotechnique, vol. 27, $n^{\circ}$ 3, 1977.

Baquelin F. Jézéquel J.F. Shields D.H. The pressuremeter and foundation engineering. Series on rock and soll mechanics, vol, $2(1974 / 1977), n^{\circ} 4$.

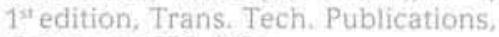
Germany, 1978, $615 p$

Baguelin F., Frank R., Jézéquel J.F. - uThe Menard pressuremeter test and the behaviour of laterally loaded piles in sand . Proceed. of ISP3 the 3 rid International Symposium on Pressuremeters, Oxford University, 2-6 April 1990. p. 381-391.

Bangratz R., Frank R, - PILATE: Programme de calcul d'un pieu isolé soumis à des efforts de flexion en tête et à des poussées latérales du sol. Notice d'utilisation, version janvier 1985.

Beikae M. Pyke R, - u A new solution for the resistance of single piles to lateral load x. Laterally loaded deep founda. tions-Analvsis and performance. ASTM Special Technical Publications, $n^{\circ} 835$. 1984, p. 21-34.

Belkhir S. Mezazigh S. Levacher D.«Non-Linear beahvior of Laterally Loaded Pile Taking into Account the Shear Stress at the Sand in. ASTM Geotechnical Testing Journal, vol. 22, $n^{\circ} 4$. December 1999, p. 308-316.

Bouafia A. - Modélisation des pieux chargés latéralement en centrifugeuse. Thèse de docteur en génie civil, Ecole centrale de Nantes, décembre 1990 , $267 \mathrm{p}$

Bouafia A., Garnier J. - «Experimental study of P-Y curves for piles in sand $n$. Proceed of the International conference CENTRIFUGE' 91, Boulder, Colorado, A.A. Balkema, 1991, p. 261-268.

Bouafia A., Garnier J., Levacher D. " Comportement d'un pleu isolé chargé latéralement dans le sable j. Comptes rendus du colloque international Fondations profondes, Presses des ponts et chaussées, 1991, p. 129-136.

Bouafia A., Bouquerra A. - « Modélisation en centrifugeuse du comportement d'un pieu flexible chargé horizontalement à proximité d'une pente $\%$. Revue canadienne de géotechnique, vol. 32, $\mathrm{n}^{\circ} 2,1995$, p. 324-335.

Bouafia A. Bouguerra A. - « Effet de la proximité du talus sur un pieu court et rigide chargé horizontalement $₫$. Revue française de qéotechnique, $n^{\circ} 75,1996$. p. $47-56$.

Bouafia A. - «Experimental analysis of large lateral displacements of piles in centrifuge n. Proceed. of the $4^{\text {th }}$ Interna- tional conference on case histories in geotechnical engineering. St-Louis, Missouri, 8-15 March 1998

Bouafia A. - « Interpretation of full-scale horizontal pile loading tests in sand Analysis of P-Y curves $m$. Proceed. of the $9^{\text {th }}$ International Conference on Piling and Deep Foundations DFI'2002, Nice, Presses des ponts et chaussées. $2002 a$, p. 609-616.

Bouafia A. - $\alpha$ Analysis of lateral reaction modulus for piles in sand from CPT test j. Comptes rendus du Symposimu PARAM'02 Identification et Détermina tion des paramètres des sols et des roches bour les calculs géotechniques, Paris, Presses des ponts et chausseees, 2002b.

Bouafia A. - "Response of a flexible pile under lateral loads in dense sand in centrifuge 3. Proceed. of ICPMG'02 International Conference on Physical Modelling in Geotechnics. St-John's, Newfoundland, Canada, Canadian Geotechnical Society, 2002c.

Bowles J.E. - Foundation analysis and design. Mc Graw-Hill, New York. 5thed., 1997

Briaud J.L. - «Pressuremeter and foundation design m. Proceed. of IN-SITU'86 Use of in-situ tests in geotechnical engineering. Geotechnical Special Publication $n^{\circ}$ 6, S.P.Clemence, Virginia Tech. Blacksburg, 1986, p. 74-115.

Briaud J.L., Smith T D., Mever B. - "Calcul des pieux chargés iatéralement à l'aide des résultats pressiométriques n. Comptes rendus du symposium La pressiométrie et ses applications en mer, Paris, LCPC-IFP, 1982, p. 389-406

Briaud J.L., Smith T.D., Tucker L.M. - 1 A pressuremeter method for laterally loa ded piles 3. International Conference on Soil Mechanics and Foundation Engineering, San Francisco, vol. 3 , 1985, p. 1353-1356

Canèpa Y,, Depresle D., Leipp J., Smirr J.L. - Fscais de sollicitations horizontales de tubes de différents diamètres fichés dans une fosse de sable de Fontainebleau. Comptes rendus d'essais FAEF 1.15.06.6, dossier 8670, LREP, Février $1988,32 \mathrm{p}$.

Carayannacon-Trézos. Baguelin F. Frank B. - «Réaction latérale des pieux: effets die forme et effets tridimensionnels». Bulletin des LPC. n० 104, 1979.

Douglas D.J., Davis E.H. - "The move. ments of buried footings due to moment and horizontal load and the movement of anchor plates 1. . Geotechnique, vol. 14, 1964.

Dunand M. - Etude expérimentale du comportement des fondations soumises au renversement. Thèse de docteur-ingénieur. Institut de Mécanique de Gre. noble, France, 1981

Duncan J.M., Yung Chang C, - * Nonlinear analysis of stress and strain in soils $x$. Journal of Soil Mechanics and Foundations Division, Proceed. of the ASCE. vol. $96, n^{\circ}$ Sm5, September 1970, p. 1629-1654.

Frank R. - Etudes théoriques des fondations profondes et d'essais en place par autoforage dans les LPC et résultats pratiques. Rapport de recherche du LCPC. $n^{\circ}$ 128, Juin 1984

Frank R. Jézéquel JF - - La résistance latérale des pieux \$. Journées de Méca- nique des Sols des LPC, Saint-Brieuc 20-22 juin 1989, $36 \mathrm{p}$

Gambin M. - "Calculation of foundations subjected to horizontal forces using pressuremeter data n. Sols-Soils, $n^{\circ} 30$ 31,1979, p. $17-59$

Garassino A - «Soil modulus for laterally loaded piles. $x$ Proceed. of the $4^{\text {th }}$ European Conference on Soil Mechanics and Foundations Enqineering, Vienna, 1976

Georgiadis M., Anagnastopoulos C., Saflekou $\mathrm{S}$. - is Centrifucal testing of lateraliv loaded piles 1). Revue canadienne de géotechnique, $n^{\circ} 259,1992$, p. 208-216

Jézécuel J.F. - Résistance latérale des pieux Le Rheu-Prévision du comportement des pieux. Rapport préliminaire aux essais, FAER, 1.05.01.7. LRPC de Saint Brieuc, janvier 1988, $43 \mathrm{p}$.

Ménard L., Gambin M., Bourdon G. "Méthode générale de calcul d'ur rideau ou pieu sollicité horizontalement en fonction des résultats pressiométriques v. Sols/Soils, vol. 1, n ${ }^{\circ} 20 / 23$ 1969. p. 16-28.

Ménard L. - $\alpha$ Le tassement des fondations et les techniques pressiométriques. Bilan après 10 ans de résultats expé rimentaux $x$. Annales de I'ITBTP. décembre 1971.

Olham D. - Lateral loads tests on piles. Thèse de master de l'université de Manchester, avril 1983.

Poulos H.G. $-\propto$ Behaviour of laterally loaded piles: 1-Single piles n. Proceed. of the American Society of Civil Engineers vol. $97, n^{\circ}$ Sm5, May 1971, p. 711-731.

Poulos H.G. Carter J.P., Small J.C. "Foundations and retaining structures-Research and practice n. Report to the $15^{t}$ international conference on soil mechanics and geotechnical engineering, vol, 4, 27-31 August 2001 pp. $2527-2606$

Reese L., Matlock H. - "Non dimensional solutions for laterally loaded piles with soil modulus assumed proportional to depth 1 . Proceed. of the 8th Texas conference on Soil Mechanics and Foundations Engineering, Austin, September 1956.

Reese L.C. - $\alpha$ The analysis of piles under lateral loadin 10. Proceed. of the Symposium Interaction structure-foundation. University of Birmingham, 1971

Robertson P.K. Hughes IM.O. Campanella R.G.. Sy A. - a Design of laterally loaded displacement piles using a driven pressuremetr $\%$, Laterally loaded deep foundations-Analysis and performance, ASTM Special Technical Publications, n' 835; 1984, p. 229-238.

Robertson P.K., Campanella R.G., Brown P.T. $-\alpha$ Design of axially and laterally loaded piles using in situ tests: a case history $)$. Revue canadienne de géotechnique, vol. 22, 1985, p. 518-527.

Ruesta P.F. Townsend F.C. - «Evaluation of laterally loaded pile group at Roosevelt bridge m. Journal of Geotechnical and Geoenvironmental Enqineering, vol. 123 $n^{\circ} 12$. December 1997, p. 1153-1161.

Smith T.D. - « Friction mobilisation F-Y curves for laterally loaded piles from the pressuremeter '). Proceed, of the International Symposium on Predictions and Performance in Geotechnical Engineering, Calgary, Canada, A.A. Balkema, 17-19 June 1987, p. 89-95. 